\title{
Magnetic Resonance Fingerprinting Review Part 2: Technique and Directions
}

\author{
Debra F. McGivney, PhD, ${ }^{1}$ ๑ Rasim Boyacıoğlu, PhD, ${ }^{1}$ Yun Jiang, PhD, ${ }^{1,5}$

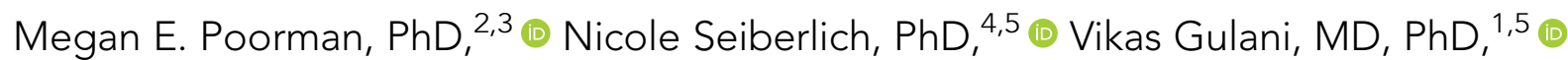

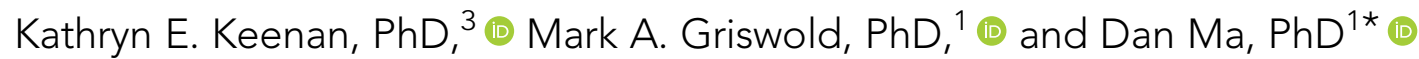

Magnetic resonance fingerprinting (MRF) is a general framework to quantify multiple MR-sensitive tissue properties with a single acquisition. There have been numerous advances in MRF in the years since its inception. In this work we highlight some of the recent technical developments in MRF, focusing on sequence optimization, modifications for reconstruction and pattern matching, new methods for partial volume analysis, and applications of machine and deep learning.

Level of Evidence: 2

Technical Efficacy: Stage 2

J. MAGN. RESON. IMAGING 2020;51:993-1007.

$\mathrm{M}$ AGNETIC RESONANCE FINGERPRINTING $(\mathrm{MRF})^{1}$ was introduced as a novel quantitative magnetic resonance imaging (MRI) technique, which is used to generate maps of MR-related tissue properties using a single acquisition. The inception of MRF has sparked numerous research projects in the MR community, ranging from repeatability and clinical applications to sequence design and reconstruction. Indeed, it was only a few years since the publication ${ }^{1}$ that review articles were written ${ }^{2,3}$ to summarize the many improvements and extensions that had been made to MRF. In this work we focus on the technical developments made to the MRF framework, specifically in terms of optimization, reconstruction, partial volume, and machine learning. Both optimization and machine learning are active research areas in their own right, and the techniques themselves are constantly evolving.

To appreciate many of the recent developments in MRF, it is imperative to understand the proposed framework for MRF from the initial works, ${ }^{1,4-6}$ and how this approach is different from conventional methods. Quantitative mapping in MRI generally involves a long acquisition in which one tissue property is mapped at a time. The signal models to quantify $T_{1}$ or $T_{2}$ are typically described using 1) exponential models of signal recovery or decay (eg, inversion recovery for $\mathrm{T}_{1},{ }^{7}$ and Carr-Purcell-
Meiboom-Gill (CPMG) for $\left.\mathrm{T}_{2}{ }^{8,9} ; 2\right)$ steady state signal models (eg, variable flip angle FLASH ${ }^{10}$ and $\mathrm{DESPOT}_{1}{ }^{11}$ for $\mathrm{T}_{1}$, $\mathrm{DESPOT}_{2},{ }^{11}$ partial spoiling steady-state free precession (SSFP), ${ }^{12}$ and double echo at steady state (DESS), ${ }^{13}$ for $\mathrm{T}_{2}$; or 3 ) from driven equilibrium or transient state of the steady state sequences (eg, Look-Locker). ${ }^{14}$ There have been many approaches that propose to quantify multiple tissue properties simultaneously using a more complex signal model. Such approaches, which use one acquisition to quantify multiple properties, include inversion recovery True-FISP ${ }^{15,16}$ for $T_{1}, T_{2}$, and proton density and QRAPMASTER ${ }^{17}$ to quantify $T_{1}, T_{2}$, proton density, and B1 field amplitude. Other quantitative methods for multiple properties include MRF spin tomography in the time domain ${ }^{18}$ to quantify $T_{1}, T_{2}$, and $B 1$, and the multipathway multiecho imaging method ${ }^{19}$ for $3 \mathrm{D}$ quantification of $\mathrm{T}_{1}, \mathrm{~T}_{2}, \mathrm{~T}_{2}{ }^{*}, \mathrm{~B} 0$, and $\mathrm{B} 1$. Quantification of additional properties, including $\mathrm{T}_{1}, \mathrm{~T}_{2} *$, and magnetic susceptibility was demonstrated, ${ }^{20}$ and magnetization transfer was quantified along with $\mathrm{R} 1$ and $\mathrm{R} 2 .^{21}$

MRF recognizes that modern computation allows for much more complex signal models, which can provide higherquality mapping than previous methods. ${ }^{1}$ As such, it typically relies on a variable acquisition scheme to generate pixelwise signal 
evolutions that are unique and distinct from the exponential recovery curves typically used in $T_{1}$ or $T_{2}$ mapping. In MRF, multiple tissue properties are quantified using a single scan, eliminating the need for registration between multiple, long acquisitions. Coupled with the variable sequence parameters, the data are typically highly undersampled in the Fourier domain, resulting in an accelerated acquisition; however, this acceleration also leads to signal evolutions which are heavily corrupted by aliasing artifacts. Instead of fitting these acquired pixel signal evolutions to an exponential model, pattern matching with a predefined dictionary of simulated signal evolutions is typically used and has been shown to be an efficient and accurate method to determine properties such as $T_{1}$ and $T_{2}$ relaxation times. ${ }^{1,5}$

The variable excitation and sampling patterns that are so important in MRF are also not unique to it. Variable repetition times were previously used in balanced SSFP (bSSFP) imaging to improve the frequency response and reduce banding artifacts, ${ }^{22,23}$ and simulated annealing was used to optimize the repetition time for this case. ${ }^{24}$ Randomized sampling was previously used, ${ }^{25,26}$ and is in the spirit of the idea of compressed sensing for MRI. ${ }^{27}$ Earlier works, ${ }^{28,29}$ and later, ${ }^{30}$ utilized randomized excitation for nuclear magnetic resonance (NMR) spectroscopy. MRF is unique in that the sequence parameters and sampling trajectories are varied together to create spatial and temporal incoherence in the signal evolutions.

Although initially implemented as a 2D acquisition, MRF was quickly modified for both for simultaneous multislice acquisitions $^{31-33}$ and $3 \mathrm{D}$ excitations, ${ }^{6,34,35}$ to obtain volumetric coverage in the brain. Example $T_{1}$ and $T_{2}$ maps from simultaneous multislice and 3D MRF acquisitions are shown in Fig. 1. Additionally, sequences have been modified for areas beyond neuro, including applications in the abdomen, ${ }^{36}$ breast, ${ }^{37,38}$ prostate, ${ }^{39}$ cardiac, ${ }^{40-42}$ knee and hip, ${ }^{43,44}$ among others. Examples from several of these works are presented in Fig. 2.

This work is the second part of a two-part review on MRF. Part $\mathrm{I}^{45}$ focuses in detail on the clinical applications to which MRF has been applied, along with repeatability studies for MRF and the potential challenges faced for the clinical implementation of the technique. In this part of the review, we focus on technical developments made in the field of MRF, specifically on developments related to sequence optimization, reconstruction, and partial volume quantification, as well as applications of machine learning and deep learning to MRF. Each of these new techniques look to improve some facet of the MRF framework, resulting in faster acquisition times, reduction in aliasing artifacts, dictionary compression, faster pattern matching, and better accuracy and precision.

\section{Sequence Optimization}

Besides clinical applications, much of the recent work on MRF focuses on improving the framework in some way, from optimizing the sequence structure, to improving the reconstruction performance, or simply finding ways to collect data more quickly. All of these types of improvements fall under the broad umbrella of optimization. To design an optimization problem, first it must be determined which aspect of MRF we want to improve, such as $T_{1}, T_{2}$ accuracy or precision, minimizing acquisition time, or sensitizing the sequence to additional properties. To understand which directions to take in this process, however, it is necessary to establish the goal of optimization and analyze the sources of error in the method, and which are most significant. To this end, appropriate metrics that will best predict and quantify the overall performance of a new MRF sequence should be used. These metrics should be highly correlated with the cost function used to find these optimal sequences, if not used directly as part of the cost function themselves. Since the MRF framework extends beyond sequence design, metrics and methods of analysis for each step in the process must be implemented, including those that account for sampling trajectories and undersampling factors, range and step size of dictionary tissue properties, and aspects of the reconstruction.

\section{Direct Sequence Optimization and Metrics}

There are many degrees of freedom available when optimizing MRF sequences, and thus many variables that can be optimized, including flip angle (FA) and repetition time (TR), echo time (TE), RF phase, sampling patterns, and so on. While flexibility in sequence design is a main tenant of the MRF framework, it can lead to a prohibitively large optimization problem. Designing a cost function for such a problem may not be simple, and can include factors such as variance of quantitative results, signal magnitude, or value of the inner product. However, the complexity of the cost function will affect both the optimization landscape of the problem and the computational techniques that are able to provide a solution.

Quantification of tissue properties was initially achieved in MRF using the inner product between the acquired signal evolutions and the precomputed dictionary. ${ }^{1}$ For this type of pattern matching in particular, the ideal set of dictionary entries would be orthogonal in the tissue dimension, although the idea of dictionary entries being different from each other is important in other reconstruction techniques. For easier separation between signals with different relaxation properties, the inner product between different signals should be very small or zero, even in the presence of noise or artifacts from undersampling. However, signal evolutions generated from similar relaxation properties are highly correlated, resulting in a difficult partial volume problem for tissues such as white matter and gray matter. ${ }^{46,47}$ Orthogonality would aid in separating a mixed signal in the case of a voxel containing multiple and different tissues, as we will discuss more in Section Partial Volume.

Three different metrics were tested as predictors of MRF performance in Sommer et al. ${ }^{48}$ Two metrics were related to the inner product between dictionary entries. The first of these was a local inner product measure, comparing 

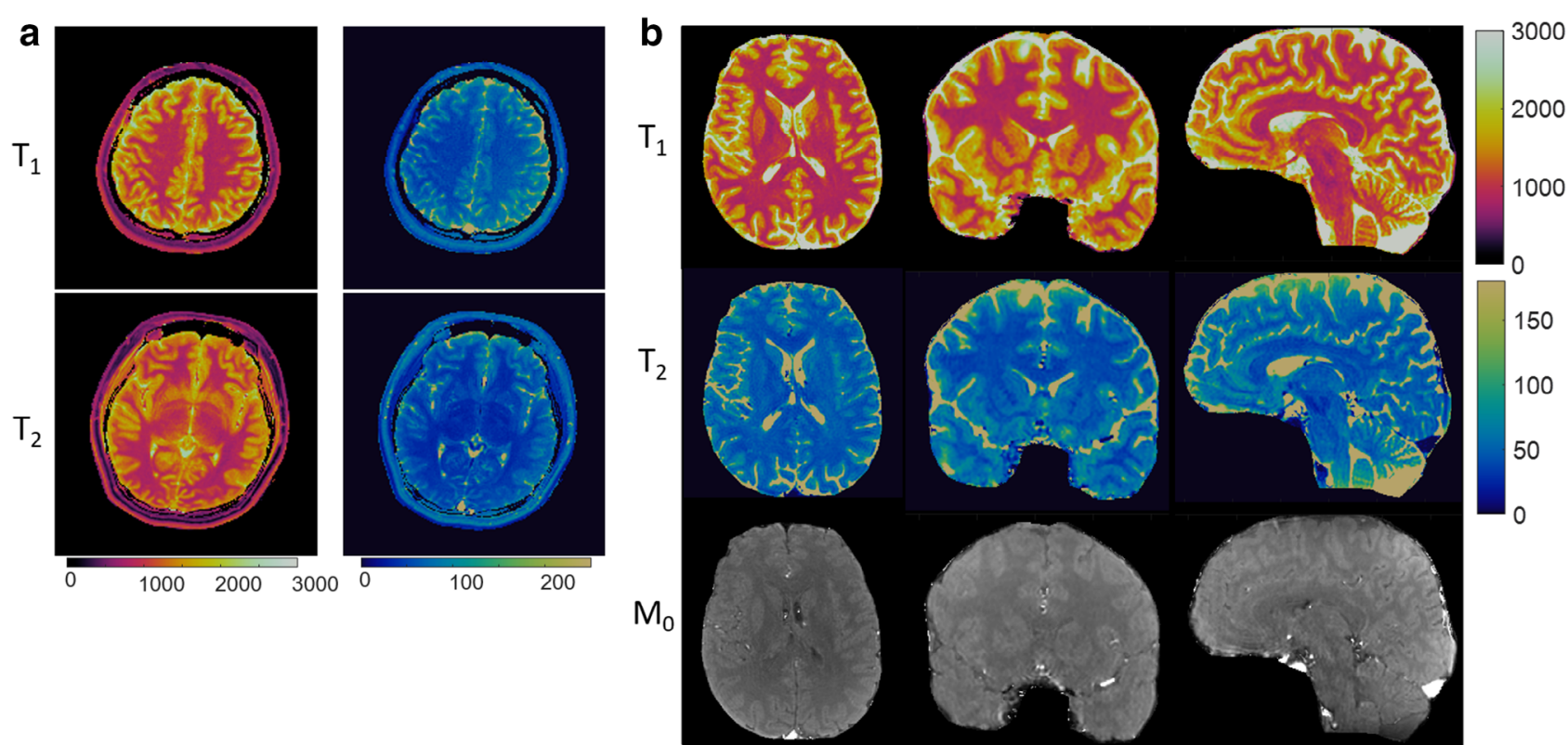

FIGURE 1: Neuro applications of MRF. Shown in (a) are 2D multislice $T_{1}, T_{2}$ maps from a normal volunteer, scanned with MRFFISP. ${ }^{5,32}$ In (b) are $T_{1}, T_{2}$, and proton density maps shown in axial, coronal, and sagittal views from three slices in a 3D MRF-FISP acquisition. ${ }^{6}$ All units for $T_{1}$ and $T_{2}$ maps are in msec. The multislice figure is reprinted with permission. ${ }^{32}$

a
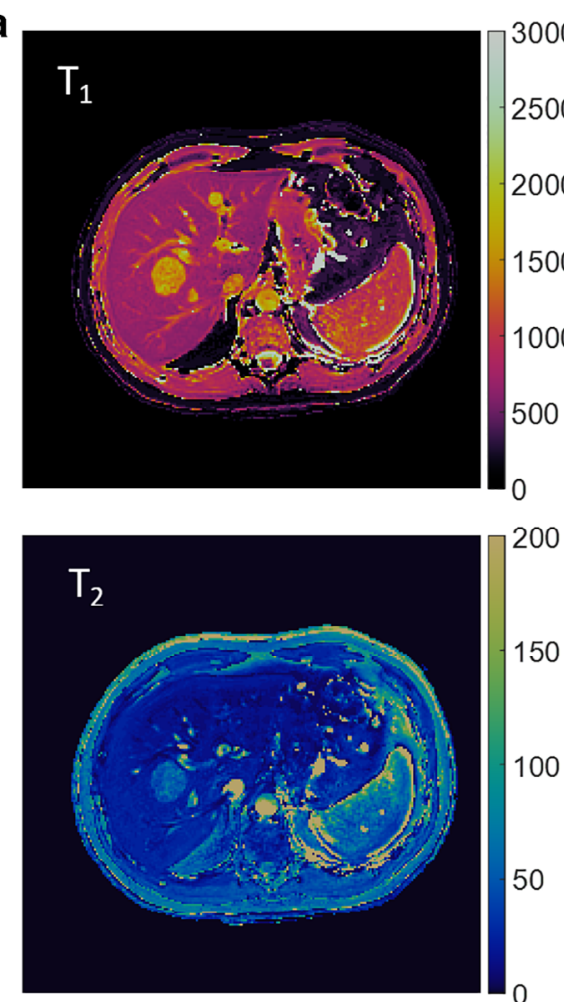

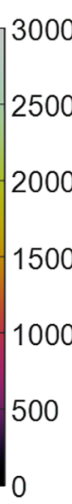

200

00 b

Apex

Mid

Base

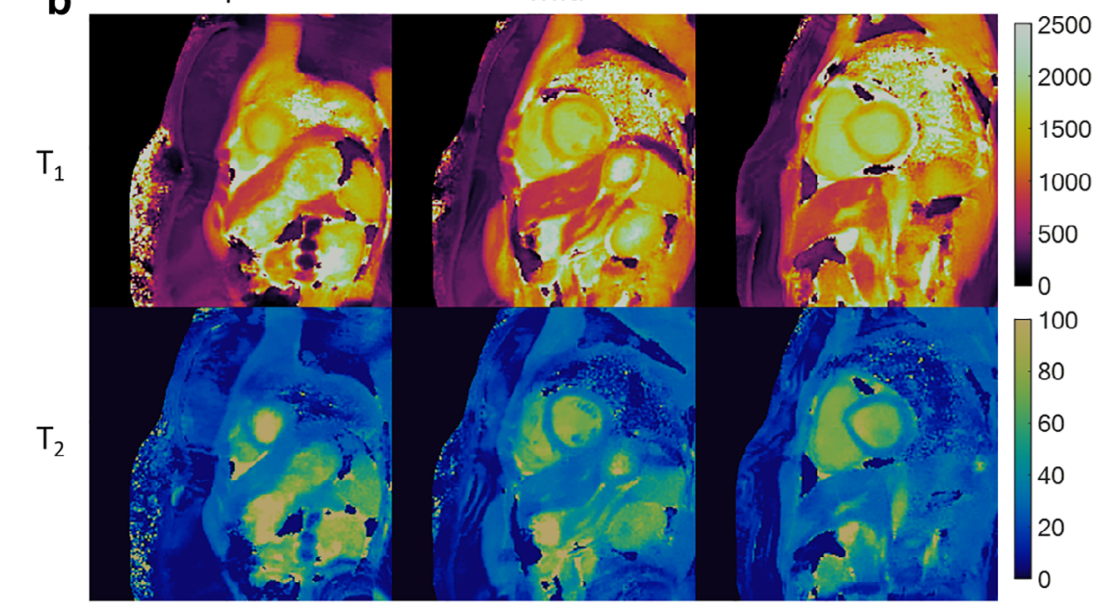

C
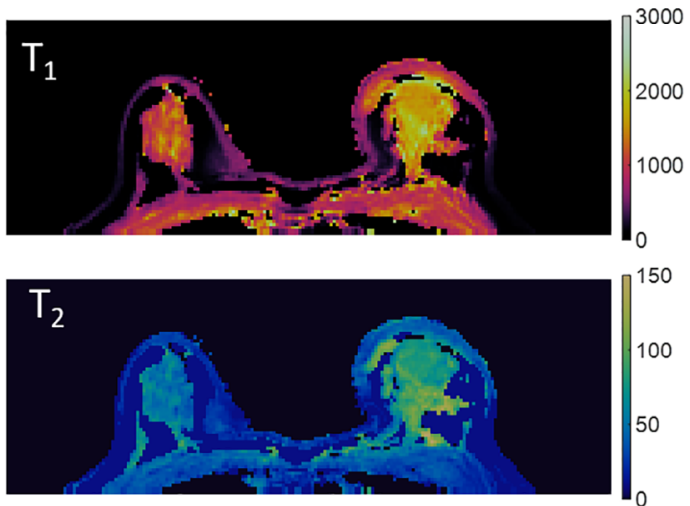

FIGURE 2: Examples of applications of MRF to different parts of the body, including (a) abdomen maps from a patient with lung adenocarcinoma metastatic to the liver using a 2D MRF scan, ${ }^{36}$ (b) cardiac multislice maps from a normal volunteer using a simultaneous multislice scan, ${ }^{41}$ and (c) breast maps from a patient with invasive ductal carcinoma in left breast using a 3D MRF scan. ${ }^{37}$ Units for all $T_{1}, T_{2}$ maps are shown in msec. Figures of abdomen and breast $T_{1}, T_{2}$ maps are reprinted with permission, with new color maps applied, from Chen et al. ${ }^{36,37}$ 
the inner product value between adjacent dictionary entries. The other was a global metric, using a wide range of dictionary entries for inner product comparison. A third metric used Monte Carlo simulations to add simulated complex Gaussian noise to dictionary signal evolutions. The inner product was then calculated to obtain the error between the ground truth $T_{1}$ and $T_{2}$ values and the computed ones. All three metrics were tested against a set of randomly generated MRF sequences. The error metric using Monte Carlo noise simulation was most successful in predicting which sequences had the best performance, as opposed to the local and global dot product metrics, and this was shown in both phantom and in vivo studies.

The inner product metric was also used in Cohen and Rosen, ${ }^{49}$ where the cost function was designed to maximize the orthogonality of the dictionary, by comparing the matrix $D^{H} D$ to the identity matrix, where $D$ is the matrix representation of the dictionary. Four different optimization techniques were examined; including 1) simulated annealing, 2) branch-and-bound, 3) interior-point, and 4) brute force, in their performance to produce an optimal sequence using this particular cost function. The interior-point algorithm produced the best sequences, in terms of scan time and $T_{2}$ accuracy. The optimal flip angle and TR patterns produced from the interior-point optimization are shown in Fig. 3, with the initialization for the interior-point algorithm shown in blue and the optimized patterns shown in red. For both the FA and TR, piecewise linear patterns were calculated from the optimization, unlike the randomized patterns used for initialization.

The Cramér-Rao bound is a statistical tool that places a lower bound on the variance of an unbiased estimator, and has recently been applied to derive optimal sequence parameters for $T_{1}$ mapping, ${ }^{50}$ and separately to increase the precision a
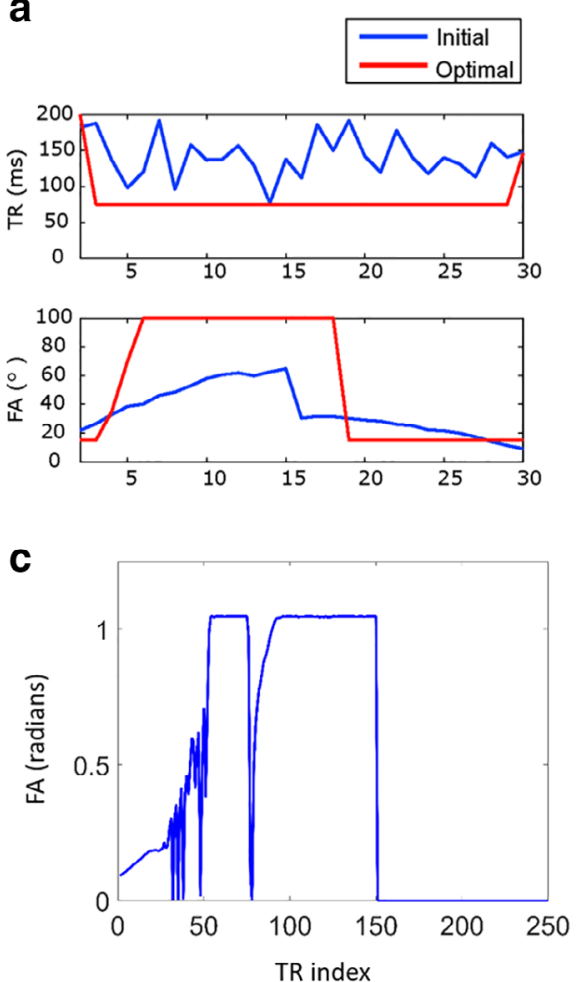
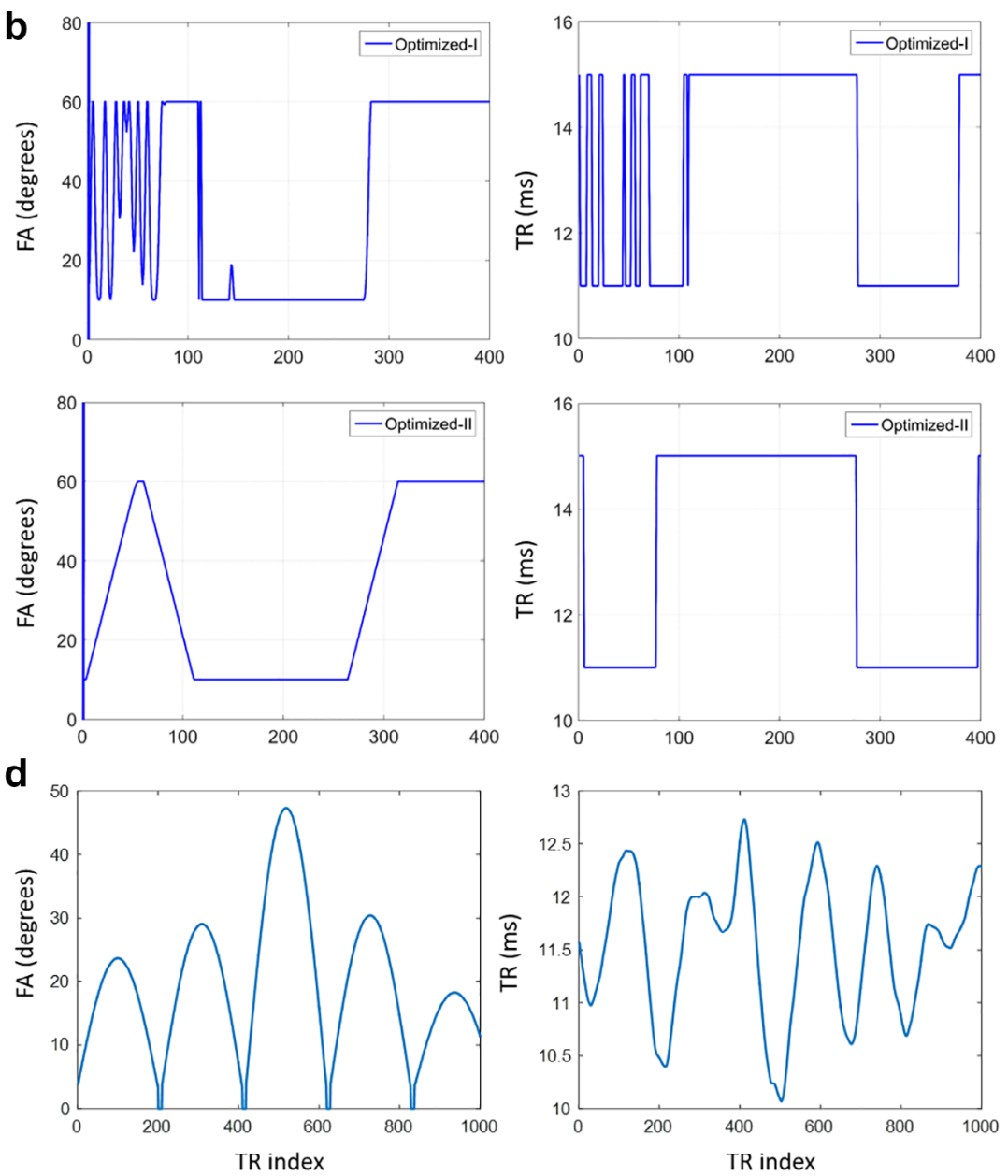

FIGURE 3: Flip angle (FA) and repetition times (TR) produced from the optimization techniques discussed in Section Sequence Optimization. The FA and TR patterns in (a) are from Cohen et $\mathrm{al}^{49}$ using the interior-point optimization, applied to a cost function that emphasizes the orthogonality of the dictionary matrix. In (b) are FA and TR patterns, ${ }^{52}$ which are based on the Cramér-Rao lower bound for unbiased estimators. "Optimized I" and "Optimized II" refer to the constraints put on the FA pattern. In Optimized I, upper and lower bounds are placed on FA and TR, whereas in in Optimized II, changes in neighboring FA values are additionally constrained. In (c) is the FA pattern from Kara et al, ${ }^{53}$ which is calculated using the genetic algorithm to optimize $T_{1}$ and $\mathrm{T}_{2}$ quality factors. The FA and TR patterns used in MRF-FISP ${ }^{5}$ are shown in (d). Figures reprinted with permission. ${ }^{49,52,53}$ 
for the relaxation values estimated with DESPOT. ${ }^{1,51} \mathrm{~A}$ rigorous derivation of a cost function to characterize the signal to noise ratio (SNR) efficiency of the MRF sequence is presented in Zhao et al, ${ }^{52}$ using the Cramér-Rao lower bound. SNR efficiency is defined in terms of the variance in the estimated tissue properties from the MRF sequence. White Gaussian noise is assumed in the derivation and the CramérRao bound is used to define a lower bound for the variance of the calculated $T_{1}$ and $T_{2}$ values using MRF. The cost function, which is the trace of the Cramér-Rao matrix, is optimized to determine FA and TR patterns that will produce optimal SNR efficiency. Two patterns are calculated, using different numbers of timepoints to vary the sequence length. In the first pattern, the constraints for the optimization include only upper and lower bounds for the FA and TR values. In the second, however, a constraint is placed on the maximum change allowed in consecutive flip angles, to force the FA pattern to be piecewise smooth. Without this additional constraint, the FA pattern produced from the optimization has rapid changes over the first hundred or so TRs, but with a constraint on the maximum flip angle change, both FA and TR patterns are structured, smoothly varying, and flat (ie, staying at either the maximum or minimum constraints) for large portions of the sequence. The FA and TR patterns from this optimization are shown in Fig. 3. The calculated FA patterns are considerably different from the sinusoidal FA pattern. ${ }^{5}$

In Kara et al, ${ }^{53}$ a cost function and optimization metric are derived in terms of a quality factor for each tissue property in MRF-FISP. The quality factors relate the variance from noise and aliasing artifacts to the variance of the computed tissue properties. By optimizing the quality factors for $T_{1}$ and $T_{2}$ simultaneously, the effects of noise and undersampling on the resulting quantitative maps can be minimized. A genetic algorithm ${ }^{54}$ is applied to produce optimal FA patterns with fixed TR, TE, and RF phase for fewer TRs than are typically used in MRF-FISP, with the resulting FA pattern shown in Fig. 3. In contrast to the FA patterns found in, ${ }^{52}$ there are no constraints placed on consecutive flip angle changes, resulting in an FA pattern with large variations and rapid changes. However, in both cases, ${ }^{52,53}$ the point is made that by rigorously optimizing the sequence structure for MRF-FISP, shorter sequences with improved efficiency may be possible than have been previously demonstrated.

Each of these approaches to MRF sequence optimization attempt to modify the current MRF framework for a measurable gain, whether it be in accuracy, precision, or efficiency, although each also focus specifically on the problem of sequence design. More recently, ${ }^{55}$ the spiral sampling patterns and spatial biases which result from the undersampling patterns commonly used in MRF were examined. Both variable and constant-density spirals were studied, each with 48 sequentially rotated spiral interleaves, and the order of the single-shot sampling was varied to determine an optimal spacing and ordering of the spirals. As opposed to a sequential ordering, $\{1,2, \ldots 48,1,2, \ldots\}$, the authors found that by using an increment of 11 for spiral ordering, that is, $\{1,12,23, \ldots\}$, shading artifacts were reduced in both $\mathrm{T}_{1}$ and $\mathrm{T}_{2}$ maps.

Other sampling trajectories for MRF have also been implemented and studied, including echo planar imaging, ${ }^{49}$ Cartesian, ${ }^{56}$ and radial $k$-space acquisitions, ${ }^{43,57}$ although optimization of the trajectory is still an open problem. Another recent work ${ }^{58}$ proposed an analytical model that includes both effects from sequence design and $k$-space sampling as an error analysis tool for MRF. This tool may be useful in assessing and predicting the performance of MRF sequences going forward. Other recent assessment and error analysis methods include error propagation analysis from nuisance parameters in quantitative $\mathrm{MR}^{59}$ and an automatic image-quality assessment. ${ }^{60}$ While neither were originally designed for MRF, they may prove to be beneficial for the MRF community.

\section{Other Improvements/Modification to the MRF sequence}

Beyond implementation of optimization algorithms to determine optimal sequence patterns, there have been numerous methods that modify existing MRF sequence structures to increase sensitivity to additional tissue properties, many of which were outlined in Part I of this review. ${ }^{45}$ However, adding tissue or system properties can complicate the quantification process; for example, this may result in extra dimensions in the dictionary. Some examples include sequences that are sensitive to $\mathrm{T}_{2}{ }^{*},{ }^{61-64}$ perfusion, ${ }^{65,66}$ and water-fat quantification. ${ }^{43,67} \mathrm{~A}$ more complicated model is needed in the case of MRF for chemical exchange, or MRF-X, ${ }^{68}$ in which six properties are quantified, including two relaxation properties to characterize two exchanging components within a voxel, volume fraction, and exchange rate.

There are still many other sequence modifications that have been made in MRF. Cardiac MRF involves modifying the sequence to an individual patient's cardiac cycle with ECG triggering, ${ }^{40}$ which necessitates a patient-specific MRF dictionary. The MRF framework was additionally modified $^{57}$ to achieve a pseudo-steady-state precession of the spins, reducing the impact from intravoxel dephasing on tissue property quantification. More recent work combines the MRF framework of chemical exchange saturation transfer (CEST) for quantification of volume fraction and exchange rate. ${ }^{69,70}$ Beyond sensitizing the sequence to in vivo tissue properties, it is possible to also quantify system properties, ${ }^{71}$ where a combination of sequence types are used to quantify $\mathrm{T}_{1}, \mathrm{~T}_{2}, \mathrm{~B} 0$, and the external $\mathrm{B} 1+$ field. $\mathrm{B} 1$ field estimation is also included in the MRF sequence. ${ }^{56}$ Adding tissue or system properties as in any of these cases can complicate the quantification process simply due to the exponential increase in dictionary size required for matching. Solutions to the problem of dictionary size are addressed in the next section.

\section{Reconstruction and Quantification}

\section{Dictionary Size and Matching Time}

Besides sequence optimization, another challenge in MRF is the size of the dictionary. When the dictionary is large, this 
can cause problems with storage and memory. Another issue is the exhaustive matching process, and when coupled with a large dictionary, can take too much time to compute. The MRF dictionary can be represented as a $2 \mathrm{D}$ matrix. The columns of this matrix represent simulated signal evolutions generated by the Bloch equations using different combinations of tissue properties, such as $T_{1}$ and $T_{2}$. The rows of the dictionary matrix are the number of timepoints, or TRs, used in the MRF sequence. Depending on the sequence type used and the granularity of the tissue property values desired, the tissue property dimension of the dictionary can easily grow from tens of thousands to millions. For example, in the case of MRF-FISP ${ }^{5}$ the sequence is used to quantify two properties: $T_{1}$ and $T_{2}$ relaxation times. However, in the case of MRF-bSSFP, off-resonance is another property that is quantified, increasing the size of the required dictionary. In a breast MRF study using MRF-FISP, ${ }^{37}$ a dictionary with 20,059 columns representing possible $T_{1}, T_{2}$ combinations was used, whereas in a brain tumor study using MRF-bSSFP, ${ }^{72}$ the additional dimension of off-resonance increases the dictionary size to 287,709 columns. In the case where the sequence is also sensitized to quantify $\mathrm{T}_{1}, \mathrm{~T}_{2}$, off-resonance, and $\mathrm{T}_{2}{ }^{*}$, the number of columns in the dictionary was reported to be over 30 million in Wang et al, ${ }^{61}$ and 64 million in Hong et al. ${ }^{63}$

Inner product pattern matching has been shown to be accurate and robust to the high degree of aliasing artifacts due to undersampling in several of the initial MRF studies, including Ma et $\mathrm{al}^{1}$ and Jiang et al. ${ }^{5}$ Also shown in both, ${ }^{1,5}$ the number of timepoints used in the sequence will have a direct impact on the quality and accuracy of the $T_{1}$ and $T_{2}$ maps. Therefore, for the sequences in these initial studies, the number of timepoints was generally between 1000 to 3000 . Strategies to handle the time and tissue property dimensions in the dictionary can lead to both reduced storage requirements and faster matching times.

To mitigate the size of the dictionary, the singular value decomposition (SVD) was used as a compression tool to reduce the time dimension in the dictionary, ${ }^{73}$ enabling a compression in the time dimension of $80-99 \%$ by projecting the dictionary onto a subspace spanned by the first few singular vectors. In this way, after projecting the dictionary onto a low-rank subspace, the size of the dictionary is reduced in the time dimension, resulting in fewer points to compare, and the inner product matching is between $3-5$ times faster. ${ }^{73}$

This idea of projecting the dictionary onto a low-rank subspace has spread into many reconstruction algorithms that use the low-rank property of the dictionary to speed up reconstruction or mitigate effects from undersampling in the reconstruction. ${ }^{74-76}$ Additionally, the SVD can be applied prior to image reconstruction, significantly reducing the size of the reconstruction problem, and has been used, for example, in the 3D MRF reconstruction ${ }^{6}$ in which the raw $k$-space data are projected onto the SVD space. By projecting the data in this way, the reconstruction problem is reduced from $14403 \mathrm{D}$ volumes to only 25 . Computing the SVD of a large matrix can be memory-intensive, and in the case where the dictionary may be too large to efficiently store and retrieve, a randomized $\mathrm{SVD}^{77}$ approach can be applied to approximate the singular vectors of the dictionary, without needing to store the full dictionary in memory. ${ }^{78}$ Multichannel transmit MRF, also called "Plug-and-Play MRF," 43 requires a different compression scheme for the dictionary due to the multiple transmit channels used. Phase unwinding is proposed to aid in dictionary compression in this particular case, ${ }^{79}$ by reconstructing the multichannel data separately, and combining after phase correction. SVD compression can then be applied to the data.

Dictionary size is the most problematic in the tissue property domain, and this dimension will grow exponentially as the number of tissue properties that the sequence is sensitized to increases, as previously described. Since the pattern matching that is used to find the best dictionary match is exhaustive, a group matching strategy was proposed ${ }^{80}$ and was able to significantly reduce the time it takes to match acquired signal evolutions to the dictionary with minimal impact on accuracy. Reported matching times were up to 70 times faster compared with exhaustive direct matching, reducing the time from $178 \mathrm{sec}-$ onds to 2.5 seconds for MRF-bSSFP. This work on fast group matching accelerated the procedure by using correlations between entries with similar relaxation properties to create subgroups within the dictionary, reducing the search space used in the matching. Acquired pixel signal evolutions were first matched to the mean signal of each group, and subgroups were eliminated when this initial inner product value was below a fixed threshold. Grouping the dictionary does not reduce the overall number of tissue property combinations, but by performing an initial match with representative signals, the matching time was reduced. Other works have incorporated the idea of a fast search for the dictionary pattern matching, ${ }^{81}$ in which the dictionary is structured as a kdimensional tree on which an approximate nearest neighbor search can be performed. In MRF-ZOOM, ${ }^{82}$ the separability of tissue properties based on the inner product model is used to develop a fast dictionary searching algorithm to reduce the matching time.

Beyond fast matching strategies, other works have focused on reducing the number of dictionary entries required for accurate quantification of tissue properties. In Yang et $\mathrm{al}^{78}$ a coarse version of the dictionary in the tissue property dimension was used, meaning that the step size in properties such as $T_{1}$ and $T_{2}$ is relatively large. Pattern matching is first done using the coarse dictionary. The dictionary is projected to a low-rank subspace where polynomial interpolation is applied to determine more accurate $T_{1}$ and $\mathrm{T}_{2}$ values. By applying interpolation to the coarse dictionary, the discretized nature of the tissue properties can be circumvented. A similar idea was proposed, ${ }^{76}$ using linear interpolation between dictionary to overcome the dictionary step size in the quantification problem. When combining this method with compression in the time domain, the storage requirements for the dictionary are greatly reduced. 


\section{Reconstruction Techniques}

A great deal of work on MRF in recent years has focused on improving the reconstruction process, specifically on how to best transform the highly undersampled $k$-space data into the image domain or directly into quantitative tissue property maps. A direct method that is commonly used for reconstruction is the nonuniform fast Fourier transform, ${ }^{83}$ in which the non-Cartesian data are first resampled to a Cartesian grid and then the fast Fourier transform is applied. Once the data are reconstructed, pattern matching is applied, although artifacts from undersampling will still impact the matching. Most iterative algorithms for MRF attempt to reduce the effect of aliasing artifacts in the image domain, and can also have the effect of reducing the number of TRs needed for the sequence, shortening the overall time for the scan, for example. ${ }^{84,85}$

Iterative approaches for MRF solve the problem by iterating between $k$-space to enforce data consistency, and the image domain, where the reconstructed signals are projected onto the MRF dictionary. Due to the application of multiple gridding and nonuniform Fourier transform iterations, the reconstruction time for such iterative algorithms can be much greater as compared with a direct gridding and reconstruction. The iterative methods aim to solve a problem of the general form:

$$
\min _{x}\|y-F x\|+\lambda T(x)
$$

where $x$ is the reconstructed image series corresponding to the acquired $k$-space data y. The operator $F$ represents the encoding function used to transform the image series to $k$-space, the operator $T$ can represent any number of penalty functionals that act on the image series, to emphasize a desired feature in the solution, for example, a wavelet transform, or total variation, and $\lambda$ is a regularization parameter. Additionally, constraints are sometimes placed on the above problem by which the signal evolutions are projected onto the dictionary subspace for matching. This matching step can be included in the iterative process, for example to ensure data consistency, ${ }^{84}$ or completed upon convergence of the algorithm.

\section{Low-Rank Reconstructions}

Many reconstruction algorithms ${ }^{41,74-76,86}$ leverage the fact that the MRF dictionary can be compressed without significant loss of information. ${ }^{73}$ While iterative reconstruction algorithms may have advantages, they may require more sophisticated computational techniques. In Assländer et $\mathrm{al}^{75}$ the reconstruction alternates between data consistency in $k$-space, and then dictionary matching in the image domain. The SVD of the dictionary is applied in the Fourier domain to solve the problem in a low-rank subspace and improve the conditioning of the problem. Variable splitting and the alternating direction method of multipliers ${ }^{87,88}$ are applied to solve the linear problem for data consistency. These computational techniques (variable splitting and alternating direction method of multipliers) are also applied in the maximum likelihood approach. ${ }^{85}$
The low-rank subspace of the dictionary was also used by Zhao et al, ${ }^{74}$ but an additional low-rank constraint is also placed on the reconstructed time series. The reconstruction problem is approximated using linear least squares, which is then solved using the conjugate gradient algorithm. Pattern matching with the dictionary is used after convergence of the algorithm to generate the quantitative tissue property maps, with examples of in vivo results from this work shown in Fig. 4. Aliasing artifacts are significantly reduced in this reconstruction, which in turn shortened the number of timepoints required for the acquisition to as few as 700 .

Similar to the previously described approaches, a lowrank approach is proposed in Hamilton et al. ${ }^{41}$ In that work, the reconstruction is performed in the SVD space to significantly reduce the time dimension of the problem. A wavelet transform is also applied, which can have the effect of smoothing the tissue property maps. Although designed for the application of simultaneous multislice cardiac MRF, this method could be applied to a single-slice acquisition as well. Example $T_{1}$ and $T_{2}$ maps from the multislice cardiac acquisition and low-rank reconstruction are in Fig. 2.

In Mazor et $\mathrm{al}^{76}{ }^{76}$ low-rank constraint is placed on the reconstructed time series. A data consistency step is applied, similar to the iterative approach ${ }^{89}$; however, instead of forcing each pixel signal evolution to match to one dictionary entry, this constraint is relaxed, allowing a linear combination of multiple entries to fit each signal evolution.

In Lima da Cruz et al, a slightly different approach was taken. ${ }^{86}$ Similar to previous methods, SVD compression for the time domain using the dictionary is applied; however, there is a spatial low-rank assumption additionally made in the image domain. Small patches of $7 \times 7$ pixels in the reconstructed singular images are assumed to have low rank. Sparse regularization is also used by applying a wavelet transform to the singular images. Example $T_{1}$ and $T_{2}$ maps from this reconstruction technique are shown in Fig. 4.

Finally, in a unique approach to the iterative reconstruction problem, Doneva et $\mathrm{al}^{90}$ used the low-rank property of the acquired data in the $\mathrm{k}-\mathrm{t}$ domain, unlike the previous methods, which all use the low-rank property of the dictionary or reconstructed image series. The SVD is applied to a small, fully sampled calibration dataset in $k$-space, and this is used as a projection matrix to recover missing $k$-space data. An advantage of this method is that the iterations are performed only in $k$-space, eliminating the repeated gridding and Fourier transform operations, which make the algorithm computationally more efficient, with reconstruction times as low as 10 seconds.

\section{Other Reconstruction Techniques and Improvements}

Apart from the aforementioned low-rank iterative approaches, other aspects of the reconstruction have been varied to improve upon the MRF framework in various ways. One 
a
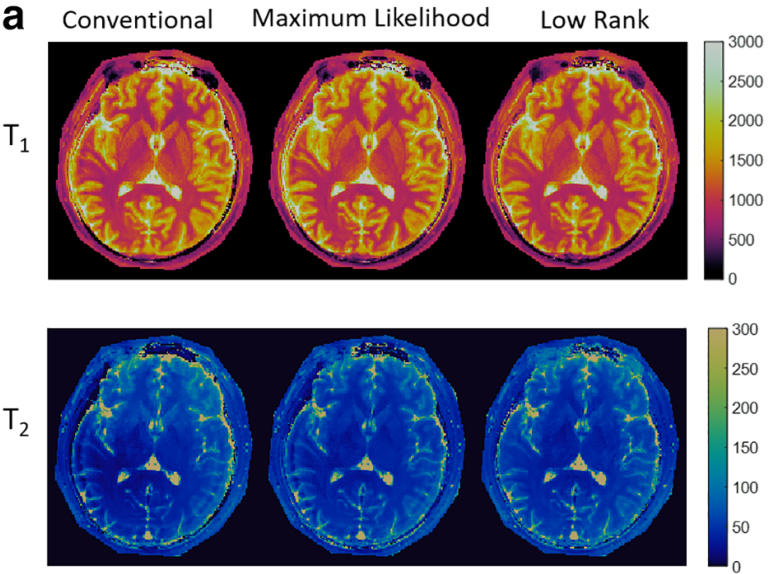
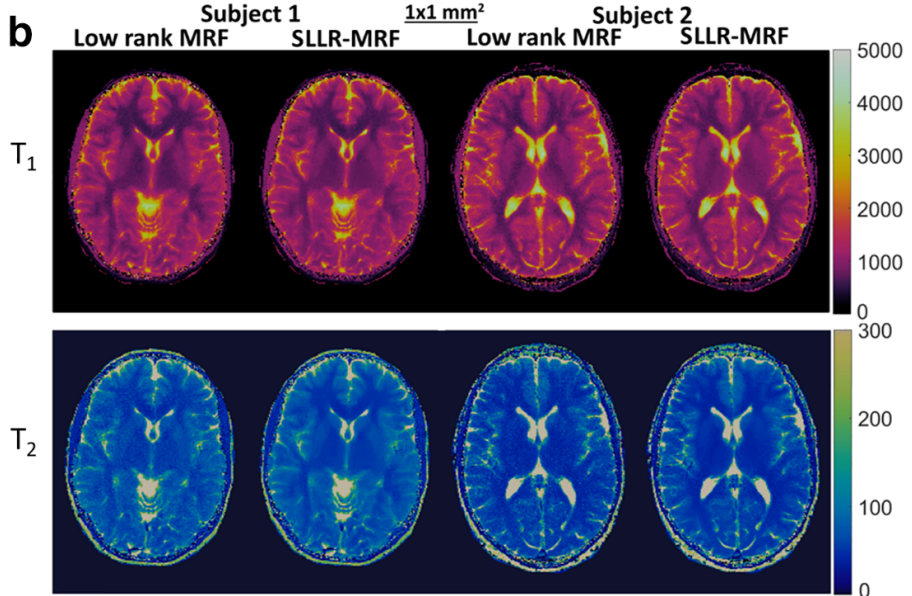

FIGURE 4: $T_{1}$ and $T_{2}$ maps obtained from reconstruction techniques outlined in Section Reconstruction and Quantification. Units for

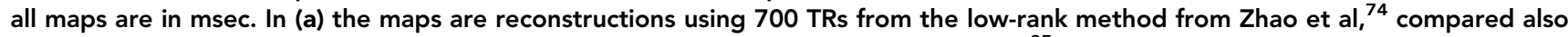
with direct reconstruction and matching, as well as the maximum likelihood approach. ${ }^{85}$ Error maps are computed by comparing to a full-sampled reconstruction as the gold standard. In (b) are maps using the sparsity and locally low rank method from Lima da Cruz et $\mathrm{al}^{86}$ shown from two different volunteers. Figures reprinted with permission, and new color maps applied. ${ }^{74,86}$

correction directly deals with blurring artifacts from B0 inhomogeneity that are a result of the accelerated spiral undersampling used in MRF. By applying a multifrequency interpolation approach to correct the MRF reconstruction, blurring is significantly reduced in MRF-FISP. ${ }^{91}$ As undersampling artifacts from a highly accelerated MRF scan can be severe, it is advantageous to develop methods that can mitigate these artifacts without sacrificing speed in the acquisition. View sharing is a technique that is used to further accelerate the acquisition by requiring fewer timepoints in the MRF sequence. ${ }^{56}$ Using a high undersampling factor, data points that are not acquired in the edges of $k$-space are filled in with those from adjacent time frames, as in key-hole acquisition. ${ }^{92}$ A similar concept is used in soft-weighted key-hole MRF, or MRF SOHO, ${ }^{33}$ in which parallel imaging, soft-gating, and the key-hole technique are combined to accelerate the scan. The sliding window reconstruction for $\mathrm{MRF}^{94}$ combines the highly undersampled frames in $k$-space to instead reconstruct fully sampled images free from aliasing artifacts. As fewer timepoints are used in the reconstruction, the MRF dictionary is modified prior to matching. Data acquisition time is reduced by up to one-third, by reducing the number of acquired timepoints from 1000 to as few as 300 .

Parameters from the reconstruction methods discussed in this section are outlined in Table 1, including reconstruction time, number of timepoints used in the acquisition, image resolution, and MRF sequence used. It is interesting to note the variation in many of these parameters; for example, reconstruction methods were performed on sequences using as few as 400 timepoints to as many as 3000 . Many works report testing on only one variant of the MRF sequence as well. Reproducibility and assessment of reconstruction techniques will be an important consideration going forward, and is discussed more in Part $1 .{ }^{45}$

\section{Partial Volume}

Partial volume can be problematic in any MR technique where the voxel size is larger than the tissue structures being imaged, which can cause blurring and degraded boundaries in the image. Many techniques have been proposed to solve the partial volume problem in $\mathrm{MRI}^{95}$; we will focus on the proposed solutions to partial volume with MRF in this section. For MRF, the unique signal evolution structure may be an advantage for partial volume; however, the problem is still illposed and difficult to solve in this context. Although partial volume is, in some sense, similar to the problem of fat/water separation, it does not necessitate a new sequence design or reconstruction processes to obtain an accurate solution. While a benefit from a direct sequence optimization may be that voxels with multiple components are more easily identified and separated, the few works on partial volume in MRF have focused finding an optimal solution to a linear inverse problem, using the MRF sequence structures that are already in place.

In $\mathrm{Ma}$ et $\mathrm{al},{ }^{1}$ a linear model was proposed to decompose the MRF pixel signal into weights corresponding to a few, predefined dictionary signal evolutions. For example, in the brain these predefined signal evolutions could correspond to white matter, gray matter, and cerebrospinal fluid (CSF). Using three representative dictionary signal evolutions, each pixel signal could be decomposed into a sum of the three, with corresponding weights, using linear least squares. An improvement on this method was made in a recent work, ${ }^{46}$ which deals specifically with how to solve this predefined linear model. As MRF signal evolutions are complex-valued, and the weights from a linear least squares model will be complex, a more realistic tissue model was proposed in the form of a partial volume dictionary, which is formed with linear combinations using only positive, real-valued weights for each 


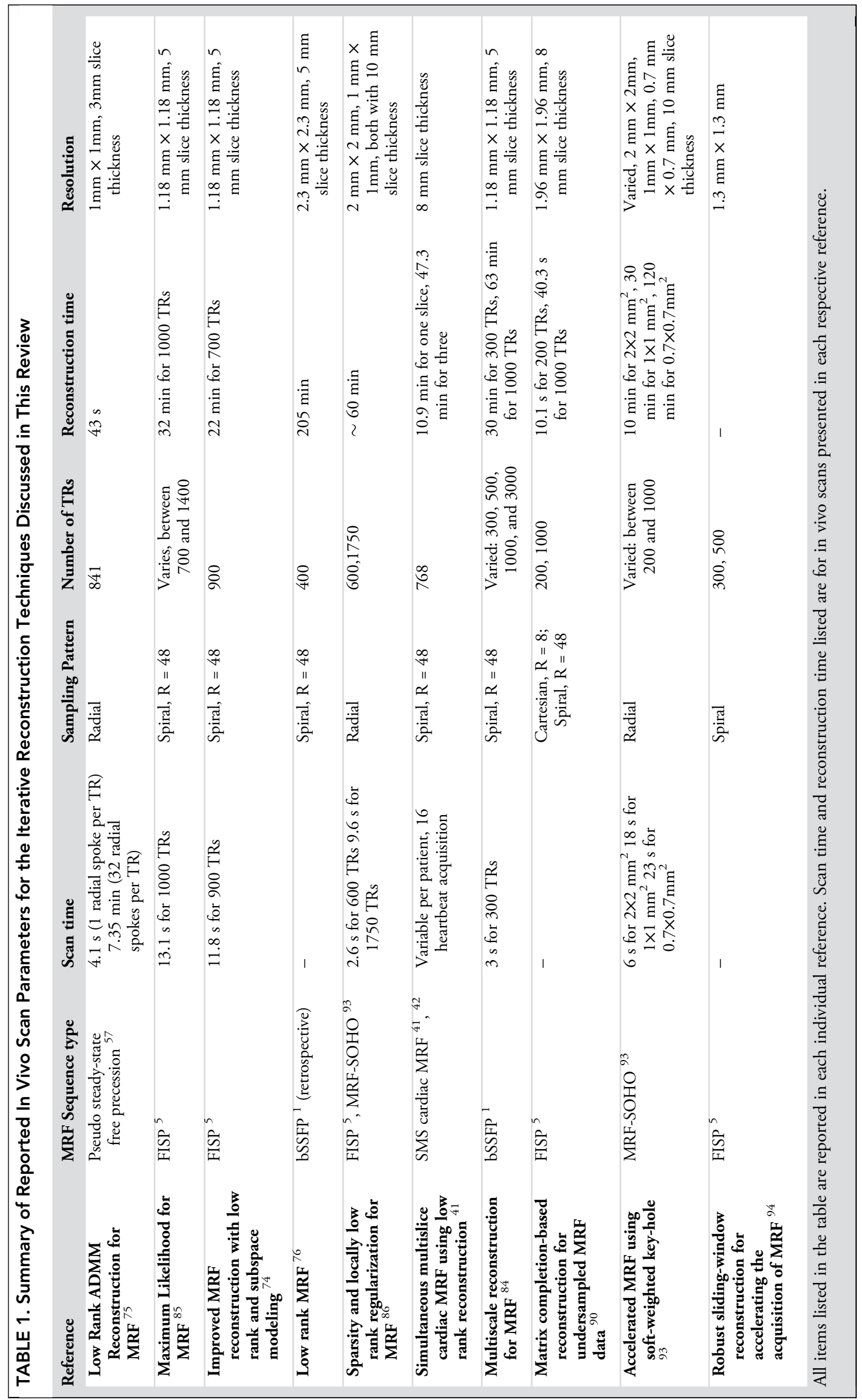


predefined tissue type. Quantification of weights is done by pattern matching with this partial volume dictionary using the inner product. Another modification included in this work is a subject-specific partial volume dictionary, which reflects the fact that there is some natural variation in the relaxation properties in the brain between subjects. ${ }^{96}$ To this end, k-means is applied to single-component MRF relaxometry maps for each subject, to determine the appropriate tissue properties to include in the partial volume dictionary. Shown in Fig. 5a,b is a comparison of the two aforementioned methods, applied to a normal volunteer, showing relative fractions of white matter, gray matter, and CSF. The method has also been applied to brain tumor patients, in which case more tissue components are used in the model to create tissue fraction maps, for example, white matter, gray matter, CSF, tumor, and peritumoral white matter.

A limitation of using a fixed tissue model, for example, assuming that brain tissue is only composed of white matter, gray matter, and CSF, may be evident in the case of pathology, where a diseased or unhealthy tissue may not be composed of these three tissues. If the diseased tissue has relaxation properties different from those represented in the model, then forcing a fixed model on the voxel signals will result in erroneous tissue fraction calculations and diseased tissue will not be properly characterized. There have been works on partial volume for MRF that remove the fixed tissue model and apply the full dictionary to mixed voxel signals. In McGivney et $\mathrm{al}^{47}$ the Bayesian paradigm for inverse problems was used to solve the problem in terms of the maximum a posterior estimator, assuming a probabilistic model for the tissue weights, and with additional postprocessing the resulting values can be combined into relative tissue fraction maps, shown in Fig. 5c for a glioblastoma brain tumor patient. The work by Tang et $\mathrm{al}^{97}$ also does not require a fixed tissue model, but instead encourages sparsity of the weight vector by using reweighted $\ell_{1}$ regularization. Although these methods are computationally more complex than the dictionary-based approach, ${ }^{46}$ they allow a more flexible tissue model when relaxation values are not known a priori, which may be the case in diseased or abnormal tissues.

\section{Applications of Machine and Deep Learning to MRF}

In recent years, machine learning and deep learning have become increasingly popular topics for research, and applications in MRI

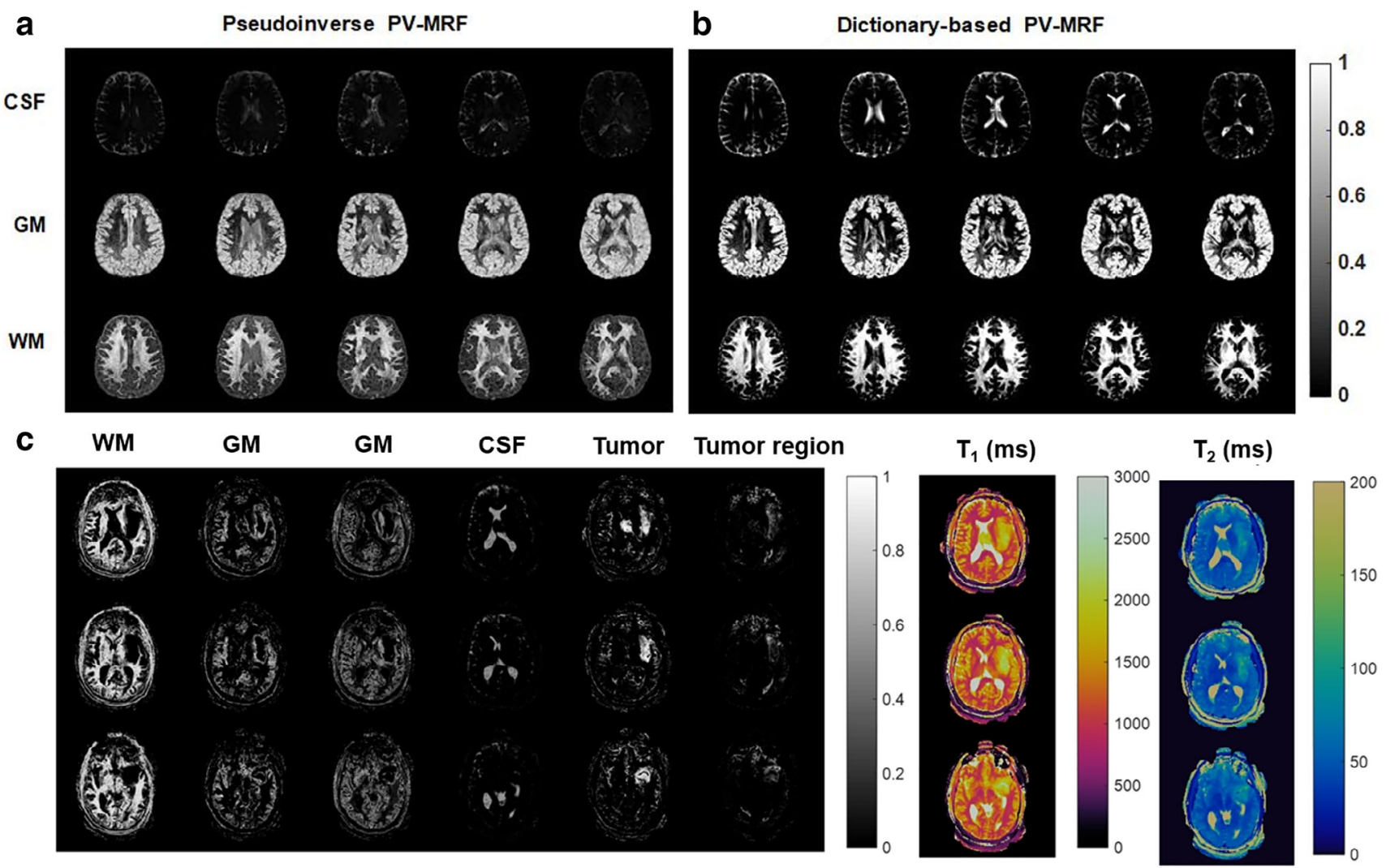

FIGURE 5: Partial volume fraction calculation from three of the discussed methods. In $(a, b)$ are fraction maps representing white matter, gray matter, and CSF in a normal volunteer, using a 3D MRF-FISP ${ }^{6}$ acquisition. The method used in part (a) is from the pseudoinverse calculation with a fixed three component dictionary, whereas in (b), linear combinations of this three component dictionary are used to generate a larger partial volume dictionary, to which pattern matching is applied. ${ }^{46}$ In (c) the method from McGivney et $\mathrm{al}^{47}$ is applied to a glioblastoma brain tumor patient. Tissues shown in this decomposition include white matter, two gray matter components, CSF, tumor, and regions surrounding the tumor. Shown on the right are the $T_{1}$ and $T_{2}$ maps obtained from 3D MRF-FISP ${ }^{6}$ in this patient. 
are frequent. ${ }^{98}$ Machine and deep learning may be a natural fit to solve some of the challenges in MRF, such as image reconstruction and pattern matching. Indeed, several of the deep-learning applications to MRF aim to either speed up the Bloch simulation calculation of a large dictionary or remove the need for a dictionary altogether by directly learning the tissue property mappings from the signal evolutions. In Yang et al, ${ }^{99}$ unsupervised learning methods were used to rapidly generate the MRF dictionary within several seconds, which could be valuable when performing sequence optimization or modification. Rapid dictionary generation was also the goal, ${ }^{100}$ where neural networks were used to generate a dictionary that is based on a patient-specific cardiac rhythm, 100 times faster than using Bloch simulation. In Boux et al, ${ }^{101}$ the relationship between the tissue property values and the dictionary is learned through regression, eliminating the exhaustive search from pattern matching. Neural networks are applied to learn tissue properties and also to directly generate synthetic qualitative images, ${ }^{102}$ bypassing the dictionary matching step. It is likely that the number of works published in this area applied to MRF will continue to grow dramatically, rapidly increasing over the coming months and years.

In the work entitled MRF-DRONE by Cohen et al, ${ }^{103}$ deep learning was applied to MRF signal evolutions, after image reconstruction, to learn the $T_{1}$ and $T_{2}$ values without direct dictionary matching. The TensorFlow framework ${ }^{104}$ was used to construct a fully connected neural network with four layers and two hidden layers, and the method was tested on both MRFEPI $^{49}$ and MRF-FISP 5 sequences. Compared with direct dictionary matching, the application of the neural network to the MRF data was between 300 to 5000 times faster. Although network training can take a considerable amount of time in these types of methods (10-74 minutes in this work), this is considered a preprocessing step that only needs to be computed once. A similar method ${ }^{105}$ trains a convolutional neural network with three layers to learn the tissue properties from a dictionary, resulting in faster quantification of $T_{1}$ and $T_{2}$ and eliminating the need to store the dictionary after training.

Another deep-learning method, named spatiallyconstrained quantification, was applied ${ }^{106}$ to learn the $T_{1}$ and $\mathrm{T}_{2}$ values directly from the MRF signal evolutions. A twostep process was used. First, the time dimension of the signal evolutions was reduced using two fully connected neural networks to learn a nonlinear mapping for feature extraction, as opposed to using SVD compression. The next step used a convolutional neural network to quantify $T_{1}$ and $T_{2}$ values at each pixel, using the spatial features of neighboring pixels calculated in the first step. $T_{1}$ and $T_{2}$ maps generated using this framework are shown in Fig. 6, using both 576 and 288 timepoints for the quantification. This framework allows a significant reduction in the MRF acquisition time, by requiring as few as one-fourth the number of acquired timepoints compared with MRF-FISP. ${ }^{5}$

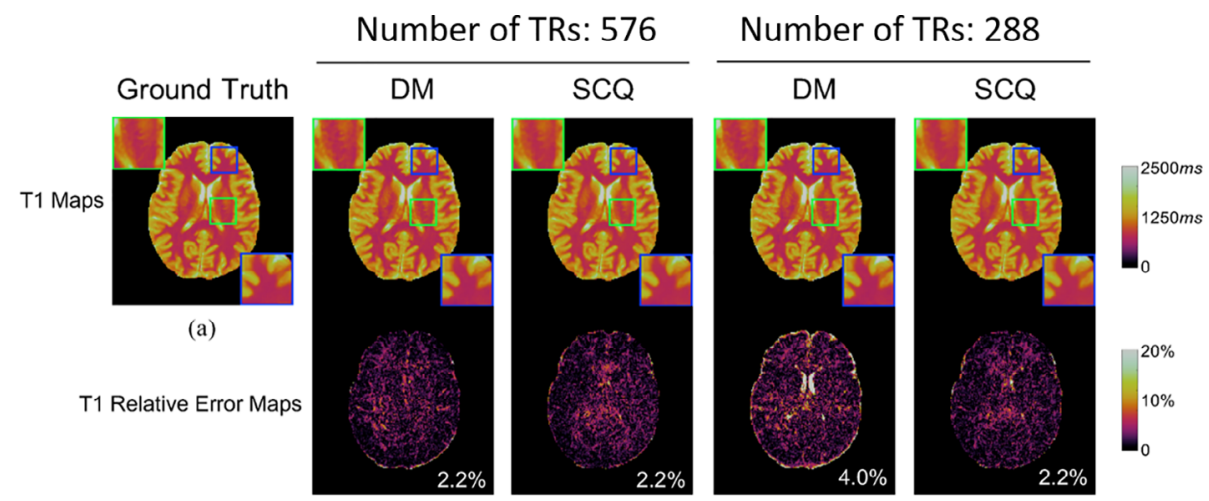

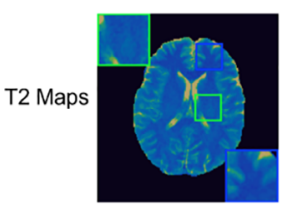

(f)

T2 Relative Error Maps

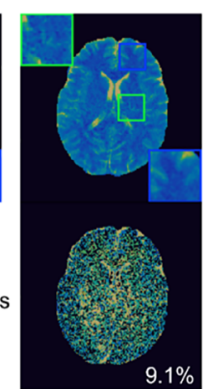

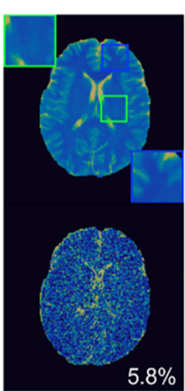
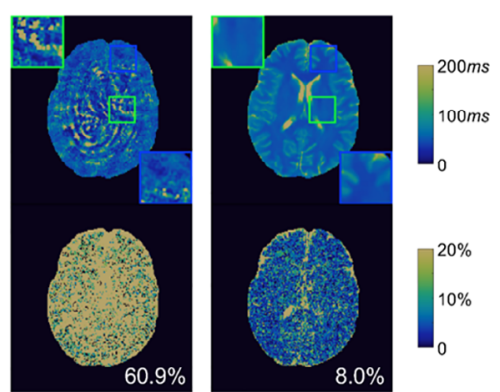

FIGURE 6: $T_{1}$ and $T_{2}$ maps generated from the deep-learning method of Fang et al. ${ }^{106}$ DM represents the results from applying direct reconstruction and pattern matching, SCQ represents the deep learning method, spatially-constrained quantification. A different number of timepoints were used, as noted in the figure. Maps are compared with an MRF with 2304 timepoints used for the ground truth, with relative error maps shown and total percent error shown in each figure. Figure reprinted with permission, and new color maps applied. ${ }^{106}$ 
One aspect to point out in the above works is the problem of the inherent complex-valued property of the MRF data. In Cohen et al, ${ }^{103}$ the absolute value of signal evolutions is taken for the input into the neural network, where as in Fang et $\mathrm{al}^{106}$ the signal evolution is split into its real and imaginary parts, resulting in a vector that is twice as long as the original. A neural network is designed, using the full complex-valued data in Virtue et al, ${ }^{107}$ specifically with the application to MRF in mind.

Although not MRF, recent works ${ }^{108,109}$ demonstrate the power of machine learning to directly quantify tissue properties from MRI data. In the case of one, ${ }^{108}$ tissue properties are learned using the MR signal model with nonlinear regression and application of a nonlinear kernel function. Application of the method allows for quantification of $T_{1}$ and $\mathrm{T}_{2}$ relaxation times using both spoiled gradient-recalled echo and dual-echo steady-state sequences. The AUTOMAP method $^{109}$ is a comprehensive deep-learning technique to replace the image reconstruction step and can be applied to various imaging methodologies to directly learn the encoding method. These works highlight the impact that machine learning can have on MRI, in particular, applying these ideas to MRF may open the door for more comprehensive optimization of the framework.

\section{Discussion}

MRF is a flexible framework that allows fast and simultaneous quantification of multiple tissue and system properties. Because the reconstruction and pattern matching do not require a particular signal shape, the framework is able to reduce the constraints on MR acquisition design and signal modeling. This flexibility can provide more rapid, robust, repeatable, and specific tissue properties for tissue characterization and clinical use. A thorough discussion of the repeatability and reproducibility of MRF, clinical applications, and potential barriers for clinical adaptation was presented in Part 1 of this review, ${ }^{45}$ which highlights the advantages and potential problems with using the MRF framework in a clinical setting. We focus our discussion here on the topics highlighted in this portion of the review, namely, the technical developments that have been made to MRF and the challenges that still remain.

The increased flexibility and degrees of freedom of MRF can pose challenges for optimization. Current studies typically optimize MRF acquisition and reconstruction separately, as evidenced in our sections on sequence optimization and reconstruction. Sequence optimization mainly focuses on improving signal separability and precision of the results, assuming perfect sampling with Gaussian white noise. Although some initial optimization methods have been studied, the global optimum for an MRF sequence design has yet to be proven. The number of degrees of freedom available in designing an MRF sequence are numerous, including sequence parameters such as FA, TR, TE, and RF phase. While sequences have been designed that optimize several of these, a comprehensive design that optimizes all of these variables simultaneously does not yet exist. MRF reconstruction methods are typically developed based on existing sequences and sampling strategies and the main goal has been to reduce image artifacts and noise. The MRF sequence design, $k$ space sampling, and reconstruction may be incorporated in a comprehensive framework for optimization in the future, and this would be a significant step in optimizing the full MRF method. However, the optimization landscape for MRF is not well understood and has not been well studied, and it is likely that the landscape is not convex and is high dimensional, and this will add additional modeling and computational complexity, as finding a global optimum is difficult with even state-of-the-art optimization techniques. Having an accurate model that can be solved using current computational methods in real time is a clear barrier for MRF to being optimized thoroughly and rapidly, for application in the clinic.

By decreasing the correlations of the signal evolutions from different tissue types, MRF may provide a unique opportunity to effectively separate multiple tissue properties from a single voxel, leading to better multiparametric mapping, partial volume separation, and microstructure characterization. For example, reducing the similarity between white matter and gray matter signal evolutions in the dictionary could result in more accurate volume fraction estimations for this common partial volume occurrence in the brain. The challenges associated with such a partial volume separation problem include multidimensional data modeling and solving inverse problems, and both of these fields are being studied and constantly evolving. An additional challenge with partial volume is that validation and establishment of a ground truth is difficult; however, the resulting multidimensional and multiscale tissue properties has the potential to make the tissue/disease characterization more specific.

There may be different metrics to assess the overall performance of the various MRF designs, including accuracy and precision of tissue property maps or total scan time, and it is likely a combination of factors such as these will need to be used. While metrics such as SNR and image quality of each individual timepoint may be applicable, their relationship to the final image quality, accuracy, and precision of the tissue property maps are nonlinear due to the pattern matching. Therefore, metrics regarding the tissue property maps are better choices for both optimization and results validation. Both phantom and in vivo validation are required for these types of metrics, and these studies have been outlined in detail in Part 1 of this review. ${ }^{45}$ Scan time is another applicable metric for clinical translation of MRF. For example, reducing the total scan time is a desirable goal for MRF, as it will reduce scanner time for patients. Current 3D MRF scan times for wholebrain coverage with $1 \mathrm{~mm}$ isotropic resolution are reported to be 7.5 minutes, ${ }^{35}$ and 5 minutes. ${ }^{34}$ Metrics that have been 
used in sequence optimization, such as accuracy or image quality, can also be used to evaluate the applications of machine and deep learning to MRF.

In addition to explicitly solving optimization problems of the MRF framework, deep learning has been implemented in reconstruction, dictionary generation, and matching steps, and has shown promising results for solving nonlinear, nonconvex, and high-dimensional problems. With the significant interest in deep learning from both engineering and clinical fields, the techniques will likely be further developed for MRF quantification, image analysis, and clinical validations.

\section{Conclusion}

MRF is a unique framework for quantitative MRI and provides multiple registered tissue property maps from a single acquisition. Recent technical developments for MRF, including sequence optimization, improved reconstruction algorithms, partial volume separation, and deep learning have been summarized in this review as important techniques to move the field of MRF forward. By developing a comprehensive optimization framework for MRF, including optimization across sequence design, reconstruction, and pattern matching, MRF will be even more widely applicable and impactful for clinical practice.

\section{Acknowledgment}

Contract grant sponsor: National Institutes of Health (NIH); Contract grant numbers: R21 EB026764 and R01 NS109439. Contract grant sponsor: Siemens Healthineers.

\section{References}

1. Ma D, Gulani V, Seiberlich N, et al. Magnetic resonance fingerprinting. Nature 2013;495:187-192.

2. Mehta BB, Coppo S, McGivney DF, et al. Magnetic resonance fingerprinting: A technical review. Magn Reson Med 2018;81:25-46.

3. Panda A, Mehta BB, Coppo S, et al. Magnetic resonance fingerprinting - An overview. Curr Opin Biomed Eng 2017;3:56-66.

4. Jiang $Y, M a D$, Jerecic $R$, et al. MR fingerprinting using the quick echo splitting NMR imaging technique. Magn Reson Med 2017;77: 979-988.

5. Jiang Y, Ma D, Seiberlich N, Gulani V, Griswold M. MR fingerprinting using fast imaging with steady state precession (FISP) with spiral readout. Magn Reson Med 2015;74:1621-1631.

6. Ma D, Jiang $Y, C$ Chen $Y$, et al. Fast $3 D$ magnetic resonance fingerprinting for a whole-brain coverage. Magn Reson Med 2018;79: 2190-2197.

7. Crawley AP, Henkelman RM. A comparison of one-shot and recovery methods in T1 imaging. Magn Reson Med 1988;7:23-34.

8. Carr H, Purcell E. Effects of diffusion on free precession in nuclear magnetic resonance experiments. Phys Rev 1954;94:630-638.

9. Meiboom S, Gill D. Modified spin-echo method for measuring nuclear relaxation times. Rev Sci Instrum 1958;29:688-691.
10. Fram EK, Herfkens RJ, Johnson GA, et al. Rapid calculation of $T 1$ using variable flip angle gradient refocused imaging. Magn Reson Imaging 1987;5:201-208.

11. Deoni SCL, Rutt BK, Peters TM. Rapid combined T1 and T2 mapping using gradient recalled acquisition in the steady state. Magn Reson Med 2003;49:515-526.

12. Bieri O, Scheffler K, Welsch GH, Trattnig S, Mamisch TC, Ganter C. Quantitative mapping of T2 using partial spoiling. Magn Reson Med 2011;66:410-418.

13. Welsch GH, Scheffler K, Mamisch TC, et al. Rapid estimation of cartilage T2 based on double echo at steady state (DESS) with 3 Tesla. Magn Reson Med 2009;62:544-549.

14. Look DC, Locker DR. Time saving in measurement of NMR and EPR relaxation times. Rev Sci Instrum 1970;41:250-251.

15. Schmitt P, Griswold MA, Jakob PM, et al. Inversion recovery TrueFISP: Quantification of T1, T2, and spin density. Magn Reson Med 2004;51: 661-667.

16. Ehses $P$, Seiberlich $N, M a ~ D$, et al. IR TrueFISP with a golden-ratiobased radial readout: Fast quantification of $\mathrm{T} 1, \mathrm{~T} 2$, and proton density. Magn Reson Med 2013;69:71-81.

17. Warntjes JBM, Dahlqvist Leinhard O, West J, Lundberg P. Rapid magnetic resonance quantification on the brain: Optimization for clinical usage. Magn Reson Med 2008;60:320-329.

18. Sbrizzi $A$, van der Heide $O, C l o o s ~ M$, et al. Fast quantitative $M R I$ as a nonlinear tomography problem. Magn Reson Imaging 2018;46:56-63.

19. Cheng C-C, Preiswerk F, Hoge WS, Kuo T-H, Madore B. Multipathway multi-echo (MPME) imaging: All main MR parameters mapped based on a single 3D scan. Magn Reson Med 2019;81:1699-1713.

20. Metere R, Kober T, Möller HE, Schäfer A. Simultaneous quantitative MRI mapping of T1, T2* and magnetic susceptibility with Multi-Echo MP2RAGE. PLoS One 2017;12:1-28.

21. Weiskopf $N$, Suckling J, Williams G, et al. Quantitative multiparameter mapping of R1, PD*, MT, and R2* at 3T: A multi-center validation. Front Neurosci 2013;7.

22. Nayak KS, Lee HL, Hargreaves BA, Hu BS. Wideband SSFP: Alternating repetition time balanced steady state free precession with increased band spacing. Magn Reson Med 2007;58:931-938.

23. Çukur T, Nishimura DG. Multiple repetition time balanced steadystate free precession imaging. Magn Reson Med 2009;62:193-204.

24. Lee KJ, Lee H-L, Hennig J, Leupold J. Use of simulated annealing for the design of multiple repetition time balanced steady-state free precession imaging. Magn Reson Med 2012;68:220-226.

25. Scheffler K, Hennig J. Frequency resolved single-shot MR imaging using stochastic k-space trajectories. Magn Reson Med 1996;35: 569-576.

26. Haldar JP, Hernando D, Liang Z-P. Compressed-sensing MRI with random encoding. IEEE Trans Med Imaging 2011;30:893-903.

27. Lustig M, Donoho D, Pauly JM. Sparse MRI: The application of compressed sensing for rapid MR imaging. Magn Reson Med 2007;58: 1182-1195.

28. Ernst RR. Magnetic resonance with stochastic excitation. J Magn Reson 1970;3:10-27.

29. Kaiser R. Coherent spectrometry with noise signals. J Magn Reson $1970 ; 3: 28-43$.

30. Kaulisch T, Kolbe $H$, Ziessow D. Nonlinear response theory in $n D$ NMR with complex stochastic excitation. J Magn Reson Ser A 1996; 121:42-49.

31. Ye $H, M a D$, Jiang $Y$, et al. Accelerating magnetic resonance fingerprinting (MRF) using t-blipped simultaneous multislice (SMS) acquisition. Magn Reson Med 2016;75:2078-2085.

32. Jiang $Y, M a D$, Bhat $H$, et al. Use of pattern recognition for unaliasing simultaneously acquired slices in simultaneous multislice MR fingerprinting. Magn Reson Med 2017;78:1870-1876. 
33. Ye H, Cauley SF, Gagoski B, et al. Simultaneous multislice magnetic resonance fingerprinting (SMS-MRF) with direct-spiral slice-GRAPPA (ds-SG) reconstruction. Magn Reson Med 2017;77:1966-1974.

34. Cao X, Ye H, Liao C, Li Q, He H, Zhong J. Fast 3D brain MR fingerprinting based on multi-axis spiral projection trajectory. Magn Reson Med 2019;289-301.

35. Liao C, Bilgic B, Manhard MK, et al. 3D MR fingerprinting with accelerated stack-of-spirals and hybrid sliding-window and GRAPPA reconstruction. Neuroimage 2017;162:13-22.

36. Chen $Y$, Jiang $Y$, Pahwa $S$, et al. MR fingerprinting for rapid quantitative abdominal imaging. Radiology 2016;279:278-286.

37. Chen Y, Panda A, Pahwa S, et al. Three-dimensional MR fingerprinting for quantitative breast imaging. Radiology 2018;290:33-40.

38. Panda A, Chen Y, Ropella-Panagis K, et al. Repeatability and reproducibility of 3D MR fingerprinting relaxometry measurements in normal breast tissue. J Magn Reson Imaging 2019 [Epub ahead of print] doi:https://doi.org/10.1002/jmri.26717.

39. Yu AC, Badve C, Ponsky LE, et al. Development of a combined MR fingerprinting and diffusion examination for prostate cancer. Radiology $2017 ; 283: 729-738$.

40. Hamilton JI, Jiang $Y$, Chen $Y$, et al. MR fingerprinting for rapid quantification of myocardial T1, T2, and proton spin density. Magn Reson Med 2017;77:1446-1458.

41. Hamilton Jl, Jiang $Y, M a D$, et al. Simultaneous multislice cardiac magnetic resonance fingerprinting using low rank reconstruction. NMR Biomed 2019;32:e4041.

42. Hamilton Jl, Jiang $Y, M a ~ D$, et al. Investigating and reducing the effects of confounding factors for robust T1 and T2 mapping with cardiac MR fingerprinting. Magn Reson Imaging 2018;53:40-51.

43. Cloos MA, Knoll F, Zhao T, et al. Multiparametric imaging with heterogeneous radiofrequency fields. Nat Commun 2016;7:12445.

44. Cloos MA, Assländer J, Abbas B, et al. Rapid radial T1 and T2 mapping of the hip articular cartilage with magnetic resonance fingerprinting. J Magn Reson Imaging 2019 [Epub ahead of print] doi:https:// doi.org/10.1002/jmri.26615.

45. Poorman ME, Martin MN, Ma D, et al. Magnetic resonance fingerprinting. Part I: Potential uses, current challenges, and recommendations. J Magn Reson Imaging 2019 [Epub ahead of print] doi:https:// doi.org/10.1002/jmri.26836.

46. Deshmane A, McGivney DF, Ma D, et al. Partial volume mapping using magnetic resonance fingerprinting. NMR Biomed 2019;32: e4082.

47. McGivney D, Deshmane A, Jiang $Y$, et al. Bayesian estimation of multicomponent relaxation parameters in magnetic resonance fingerprinting. Magn Reson Med 2018;80:159-170.

48. Sommer K, Amthor T, Doneva M, Koken P, Meineke J, Börnert P. Towards predicting the encoding capability of MR fingerprinting sequences. Magn Reson Imaging 2017;41:7-14.

49. Cohen O, Rosen MS. Algorithm comparison for schedule optimization in MR fingerprinting. Magn Reson Imaging 2017;41:15-21.

50. Liu Y, Buck JR, Ikonomidou VN. Generalized min-max bound-based MRI pulse sequence design framework for wide-range T1 relaxometry: A case study on the tissue specific imaging sequence. PLoS One 2017;12:1-20.

51. Teixeira RPAG, Malik SJ, Hajnal JV. Joint system relaxometry (JSR) and Crámer-Rao lower bound optimization of sequence parameters: A framework for enhanced precision of DESPOT T1 and T2 estimation. Magn Reson Med 2018;79:234-245

52. Zhao B, Haldar JP, Liao C, et al. Optimal experiment design for magnetic resonance fingerprinting: Cramer-Rao bound meets spin dynamics. IEEE Trans Med Imaging 2019;38:844-861.

53. Kara D, Fan M, Hamilton J, Griswold M, Seiberlich N, Brown R. Parameter map error due to normal noise and aliasing artifacts in MR fingerprinting. Magn Reson Med 2019;81:3108-3123.
54. Davis L. Handbook of genetic algorithms. New York: Van Nostrand Reinhold; 1991.

55. Körzdörfer G, Pfeuffer J, Kluge T, Hensel B, Meyer CH, Nittka M. Effect of spiral undersampling patterns on FISP MRF parameter maps. Magn Reson Imaging 2019 [Epub ahead of print] doi:https://doi.org/ 10.1016/j.mri.2019.01.011

56. Buonincontri G, Sawiak SJ. MR fingerprinting with simultaneous B1 estimation. Magn Reson Med 2016;76:1127-1135.

57. Assländer J, Glaser SJ, Hennig J. Pseudo steady-state free precession for MR-fingerprinting. Magn Reson Med 2017;77:1151-1161.

58. Stolk CC, Sbrizzi A. Understanding the combined effect of k-space undersampling and transient states excitation in MR Fingerprinting reconstructions. IEEE Trans Med Imaging 2019 [Epub ahead of print] doi:https://doi.org/10.1109/TMI.2019.2900585.

59. Lankford $\mathrm{CL}$, Does MD. Propagation of error from parameter constraints in quantitative MRI: Example application of multiple spin echo T2 mapping. Magn Reson Med 2018;79:673-682.

60. Jang J, Bang K, Jang H, Hwang D. Quality evaluation of no-reference MR images using multidirectional filters and image statistics. Magn Reson Med 2018;80:914-924.

61. Wang CY, Coppo S, Mehta BB, Seiberlich N, Yu X, Griswold MA. Magnetic resonance fingerprinting with quadratic RF phase for measurement of $\mathrm{T} 2 *$ simultaneously with $\delta f, T 1$, and T2. Magn Reson Med 2019;81:1849-1862.

62. Wyatt CR, Smith TB, Sammi MK, Rooney WD, Guimaraes AR. Multiparametric T2* magnetic resonance fingerprinting using variable echo times. NMR Biomed 2018;31:e3951.

63. Hong T, Han D, Kim D-H. Simultaneous estimation of PD, T1, T2, T2* and $\triangle \mathrm{BO}$ using magnetic resonance fingerprinting with background gradient compensation. Magn Reson Med 2019;81:2614-2623.

64. Rieger B, Akçakaya M, Pariente JC, et al. Time efficient whole-brain coverage with MR Fingerprinting using slice-interleaved echo-planarimaging. Sci Rep 2018;8:6667.

65. Su P, Mao D, Liu P, et al. Multi-parametric estimation of brain hemodynamics with MR Fingerprinting ASL (MRF-ASL). Magn Reson Med 2017;78:1812-1823.

66. Wright $K L$, Jiang $Y, M a D$, et al. Estimation of perfusion properties with MR fingerprinting arterial spin labeling. Magn Reson Imaging 2018;50:68-77.

67. Cencini M, Biagi L, Kaggie JD, Schulte RF, Tosetti M, Buonincontri G Magnetic resonance fingerprinting with dictionary-based fat and water separation (DBFW MRF): A multi-component approach. Magn Reson Med 2019;81:3032-3045.

68. Hamilton J, Deshmane A, Griswold M, Seiberlich N. MR fingerprinting with chemical exchange (MRF-X) for in vivo multi-component relaxation and exchange rate mapping. In: Proc 24th Annual Meeting ISMRM, Singapore; 2016. p 431.

69. Cohen O, Huang S, McMahon MT, Rosen MS, Farrar CT. Rapid and quantitative chemical exchange saturation transfer (CEST) imaging with magnetic resonance fingerprinting (MRF). Magn Reson Med 2018:80:2449-2463.

70. Zhou Z, Han P, Zhou B, et al. Chemical exchange saturation transfer fingerprinting for exchange rate quantification. Magn Reson Med 2018;80:1352-1363.

71. Körzdörfer $G$, Jiang $Y$, Speier $P$, et al. Magnetic resonance field fingerprinting. Magn Reson Med 2019;81:2347-2359.

72. Badve C, Yu A, Dastmalchian S, et al. MR fingerprinting of adult brain tumors: Initial experience. Am J Neuroradiol 2017;38:492-499.

73. McGivney DF, Pierre E, Ma D, et al. SVD compression for magnetic resonance fingerprinting in the time domain. IEEE Trans Med Imaging 2014;33:2311-2322.

74. Zhao B, Setsompop K, Adalsteinsson E, et al. Improved magnetic resonance fingerprinting reconstruction with low-rank and subspace modeling. Magn Reson Med 2018;79:933-942. 
75. Assländer J, Cloos MA, Knoll F, Sodickson DK, Hennig J, Lattanzi R Low rank alternating direction method of multipliers reconstruction for MR fingerprinting. Magn Reson Med 2018;79:83-96.

76. Mazor G, Weizman L, Tal A, Eldar YC. Low-rank magnetic resonance fingerprinting. Med Phys 2018;45:4066-4084.

77. Halko N, Martinsson P-G, Shkolnisky Y, Tygert M. An algorithm for the principal component analysis of large data sets. SIAM J Sci Comput $2011 ; 33: 2580-2594$

78. Yang $M, M a D$, Jiang $Y$, et al. Low rank approximation methods for MR fingerprinting with large scale dictionaries. Magn Reson Med 2018;79:2392-2400

79. Lattanzi R, Zhang B, Knoll F, Assländer J, Cloos MA. Phase unwinding for dictionary compression with multiple channel transmission in magnetic resonance fingerprinting. Magn Reson Imaging 2018;49:32-38.

80. Cauley SF, Setsompop K, Ma D, et al. Fast group matching for MR fingerprinting reconstruction. Magn Reson Med 2015;74:523-528.

81. Cline CC, Chen X, Mailhe B, et al. AIR-MRF: Accelerated iterative reconstruction for magnetic resonance fingerprinting. Magn Reson Imaging 2017;41:29-40.

82. Wang Z, Zhang J, Cui D, et al. Magnetic resonance fingerprinting using a fast dictionary searching algorithm: MRF-ZOOM. IEEE Trans Biomed Eng 2018 [Epub ahead of print] doi:0.110.

83. Fessler JA, Sutton BP. Nonuniform fast Fourier transforms using minmax interpolation. IEEE Trans Signal Process 2003;51:560-574.

84. Pierre EY, Ma D, Chen Y, Badve C, Griswold MA. Multiscale reconstruction for MR fingerprinting. Magn Reson Med 2016;75: 2481-2492.

85. Zhao B, Setsompop K, Ye H, Cauley SF, Wald LL. Maximum likelihood reconstruction for magnetic resonance fingeprinting. IEEE Trans Med Imaging 2016;35:1812-1823.

86. Lima da Cruz G, Bustin A, Jaubert O, Schneider T, Botnar RM Prieto C. Sparsity and locally low rank regularization for MR fingerprinting. Magn Reson Med 2019;81:3530-3543.

87. Yang J, Zhang Y. Alternating direction algorithms for 11-prolbmes in compressive sensing. SIAM J Sci Comput 2011;33:250-278.

88. Boyd S, Parikh N, Chu E, Peleato B, Eckstein J. Distributed optimization and statistical learning via the alternating direction method of multipliers. Found Trends Mach Learn 2010;3:1-122.

89. Davies M, Puy G, Vandergheynst P, Wiaux Y. A compressed sensing framework for magnetic resonance fingerprinting. SIAM J Imaging Sci 2014;7:2623-2656.

90. Doneva M, Amthor T, Koken P, Sommer K, Börnert P. Matrix completion-based reconstruction for undersampled magnetic resonance fingerprinting data. Magn Reson Imaging 2017;41:41-52.

91. Ostenson J, Robison RK, Zwart NR, Welch EB. Multi-frequency interpolation in spiral magnetic resonance fingerprinting for correction of off-resonance blurring. Magn Reson Imaging 2017;41:63-72.

92. Van Vaals JJ, Brummer ME, Dixon WT, et al. "Key-hole" method for accelerating imaging of contrast agent uptake. J Magn Reson Imaging 1993;3:671-675.
93. Cruz G, Schneider T, Bruijnen T, Gaspar AS, Botnar RM, Prieto C. Accelerated magnetic resonance fingerprinting using soft-weighted key-hole. PLoS One 2018;13:e0201808.

94. Cao X, Liao C, Wang Z, et al. Robust sliding-window reconstruction for Accelerating the acquisition of MR fingerprinting. Magn Reson Med 2017;78:1579-1588.

95. Tohka J. Partial volume effect modeling for segmentation and tissue classification of brain magnetic resonance images: A review. World J Radiol 2014;6:855-864.

96. Badve $\mathrm{C}, \mathrm{Yu}$ A, Rogers $\mathrm{M}$, et al. Simultaneous T1 and T2 brain relaxometry in asymptomatic volunteers using magnetic resonance fingerprinting. Tomography 2015;1:136-144.

97. Tang S, Fernandez-Granda C, Lannuzel S, et al. Multicompartment magnetic resonance fingerprinting. Inverse Probl 2018;34:094005.

98. Mazurowski MA, Buda M, Saha A, Bashir MR. Deep learning in radiology: An overview of the concepts and a survey of the state of the art with focus on MRI. J Magn Reson Imaging 2019;49:939-954.

99. Yang M, Jiang Y, Ma D, Mehta B, Griswold M. Game of learning Bloch equation simulations for MR fingerprinting. In: Proc 26th Annual Meeting ISMRM, Paris; 2018. p 0673.

100. Hamilton JI, Currey D, Griswold M, Seiberlich N. A neural network for rapid generation of cardiac MR fingerprinting dictionaries with arbitrary heart rhythms. In: Proc 27th Annual Meeting ISMRM, Montreal; 2019. p 2421.

101. Boux F, Forbes F, Arbel J, Barbier EL. Dictionary-free MR fingerprinting parameter estimation via inverse regression. In: Proc 26th Annual Meeting ISMRM, Paris; 2018. p 4259.

102. Virtue P, Tamir JI, Doneva M, Yu SX, Lustig M. Learning Contrast Synthesis from MR Fingerprinting. In: Proc 26th Annual Meeting ISMRM, Paris; 2018. p 0676.

103. Cohen O, Zhu B, Rosen MS. MR fingerprinting deep reconstruction network (DRONE). Magn Reson Med 2018;80:885-894.

104. Abadi M, Barham P, Chen J, et al. TensorFlow: A system for largescale machine learning. In: 12th USENIX Symp Oper Syst Des Implementation, Savannah, GA; 2016;265-283.

105. Hoppe E, Körzdörfer G, Würfl T, et al. Deep learning for magnetic resonance fingerprinting: A new approach for predicting quantitative parameter values from time series. Stud Health Technol Inform 2017; 243:202-206.

106. Fang Z, Chen Y, Liu M, et al. Deep learning for fast and spatiallyconstrained tissue quantification from highly-accelerated data in magnetic resonance fingerprinting. IEEE Trans Med Imaging 2019 [Epub ahead of print] doi:https://doi.org/10.1109/TMI.2019.2899328.

107. Virtue P, Yu SX, Lustig M. Better than real: Complex-valued neural nets for MRI fingerprinting. In: 2017 IEEE Int Conf Image Process; 2017:3953-3957.

108. Nataraj G, Nielsen J-F, Scott C, Fessler JA. Dictionary-free MRI PERK: Parameter estimation via regression with kernels. IEEE Trans Med Imaging 2018;37:2103-2114.

109. Zhu B, Liu JZ, Cauley SF, Rosen BR, Rosen MS. Image reconstruction by domain-transform manifold learning. Nature 2018;555:487-492. 